\title{
Synthesis of Integral Photography Images from Raw Images Captured Using a Consumer Light Field Camera
}

\author{
Kazuhisa Yanaka, Toshiaki Yamanouchi \\ Kanagawa Institute of Technology \\ Atsugi, Kanagawa, Japan \\ yanaka@ic.kangawa-it.ac.jp; yama@ic.kangawa-it.ac.jp
}

\begin{abstract}
In this paper, we propose a new method to synthesize an integral photography image to be displayed on a flat panel display based on a raw image captured using a commercial light field camera. Among stereoscopic image display methods, Lippmann's integral photography has the advantage of producing a high sense of reality because parallax occurs in the horizontal and vertical directions. When a stereoscopic image for IP is produced via live action, a large-scale camera system is typically required. By contrast, a commercial light field camera is portable and can record the intensity and direction of incident light by stacking a microlens array on a high-definition image sensor. However, a raw image obtained from the light field camera cannot be directly used for IP. We solve this problem by introducing conversion between IP images. We develop an experimental system for synthesizing images for IP from a raw image captured using a commercial light field camera (Lytro A1). Results confirm that an autostereoscopic image with horizontal and vertical parallax is displayed when observing the image using a fly's eye lens on a PC screen. The amount of popping out or sinking can be controlled by changing the parameters of the program.
\end{abstract}

Keywords: Light field camera, Integral photography, Fly's eye lens. Extended fractional view.

\section{Introduction}

Among stereoscopic image display methods, Lippmann's [1] integral photography (IP) exhibits the advantage of producing a high sense of reality because parallax occurs in the horizontal and vertical directions. A stereoscopic image can be produced via computer graphics or live action. In the latter, a large-scale camera system is typically required.

A light field camera can record the intensity and direction of incident light by stacking a microlens array on a highdefinition image sensor, such as a complementary metal-oxide-semiconductor. In conventional cameras, focusing can only be achieved during the time of shooting. By contrast, a light field camera exhibits the advantage of being able to refocus after shooting.

In addition, a light field camera can also be used as an IP camera. However, several issues should be solved.

Accordingly, we develop a method for synthesizing images for IP from raw images captured using a commercial light field camera (Lytro).

\section{Method}

Fig. 1 (a) shows a simple imaging and display system for live action-based IP. During imaging time, the light emitted from a real 3D object (i.e. a cat in this figure) passes through the fly's eye lens of the light field camera placed at the center, enters each pixel of the image sensor, and is converted into an electric signal. A raw image is obtained by converting this electric signal into data. The black circles in the lens are the center of curvatures. That is, they are the center of the sphere when the surface of the lens is approximated by a spherical surface. A ray passing through the center of curvatures travels in a straight line, facilitating drawing.

A dedicated display is used to reproduce a 3D image. However, assume that a light field camera is used as the display. Then, light is emitted from each pixel and spreads across space through the fly's eye lens. In accordance with the principle of reversibility, light follows the same path traveled when shooting but in the opposite direction, reaching the position of the original object. However, the light goes straight further because the original object is not there. If an observer is present, then the observer feels that the light is coming from the position where the object is originally located, although 
the light is actually coming from the pixel. Thus, the observer perceives a stereoscopic image in empty space. Accordingly, an autostereoscopic display is feasible in principle. However, this method suffers from several problems.

First, a light field camera captures only the light emitted from the surface of an actual object facing it. At the time of display, however, the image is viewed from the opposite direction; thus, an unnatural image in which depth is reversed is seen, similar to a mask viewed from the back side. This problem can be solved by flipping pixels vertically and horizontally within each element image. In such case, however, all the 3D objects to be displayed are submerged into the other side of the display surface plane, such that no 3D object pops out to this side.

Second, the number of minute convex lenses constituting the fly's eye lens in the vertical and horizontal directions must be the same at the times of imaging and display. That is, the fly's eye lenses of the camera and the display must be geometrically similar, although their absolute size may differ. Furthermore, the number of pixels per lens pitch must be the same. Meeting this condition is impractical unless extremely expensive custom-made parts are used.

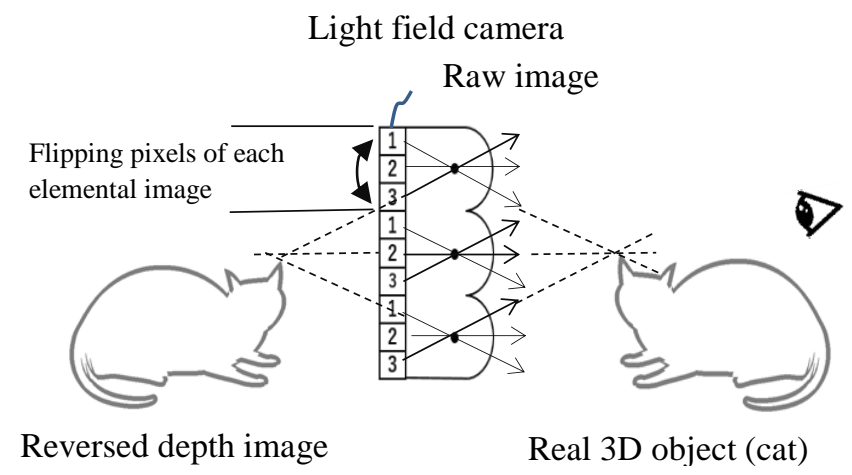

Fig.1 Creation of reversed depth image by flipping the pixels of each elemental image

To solve the aforementioned problems, we propose the method shown in Fig. 2, in which an IP display has been added. In such case, the problem is determining the pixel value of the IP image. That is, the light emitted from each pixel of the light field camera must be replaced by the light emitted from each pixel of the display at a certain distance.

Here, we focus on Pixel P of the display. The light emitted from this pixel goes straight through the nearest center of Curvature $\mathrm{C}$ and reaches Lens 3 of the light field camera. The light rays emitted from Lens 3 are Rays 1, 2, and 3. Among the three rays, Ray 1 is the most parallel to Ray P. Therefore, the value of Pixel Q, which is the source of Ray 3 , is set to Pixel P. This procedure is repeated until all the pixel values of the display are determined. The information included in the light field camera is transferred to the display using this procedure.

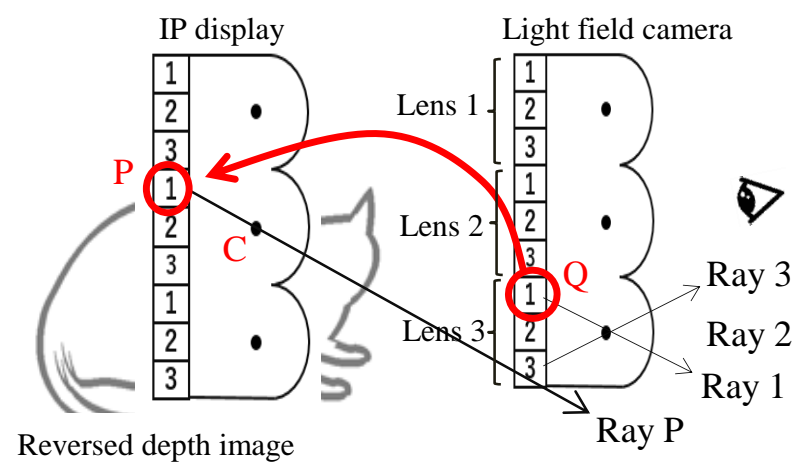

Fig. 2 Conversion from the pixels of the original position (camera) to those of the different position (display) 
Thereafter, the light field camera is no longer necessary, as shown in Fig. 1 (c). For clarity, the number of pixels per convex lens is an integer. That is, the integer view method is used for explanation. However, the proposed method can be applied even when the extended fractional view [2] method, in which the number of pixels per convex lens is a non-integer, is adopted.
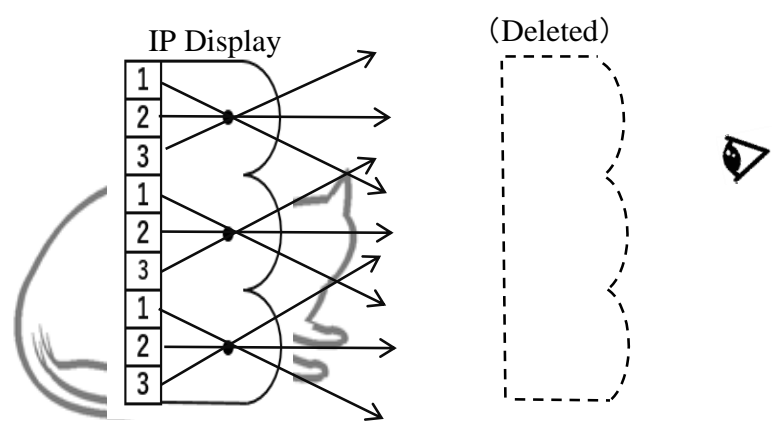

Fig, 3 Light field camera is no longer necessary.

\section{Experiment}

The camera Lytro A1 was used in the experiment. To retrieve raw data from the camera, the camera was connected to a PC via USB and an application called Lytro Desktop was runned. To determine the format of raw files, with an extension of ltr, information from a third party site [3] is useful. Nevertheless, the header and image data parts cannot be separated automatically, and thus, they were separated manually. The center positions of the lenses became clear by performing depacking, Bayer color filter processing, and translation and rotation calibration between the fly's eye lens and the image sensor. The subsequent process is described in the previous chapter. When the finally obtained IP image was displayed on the PC screen and observed through the fly's eye lens, parallax was obtained in the horizontal and vertical directions, as shown in Fig. 4, and a stereoscopic effect was achieved.

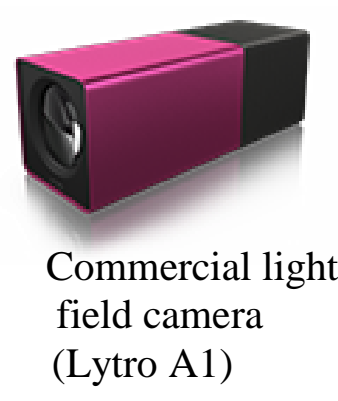

Commercial light field camera Lytro A1)

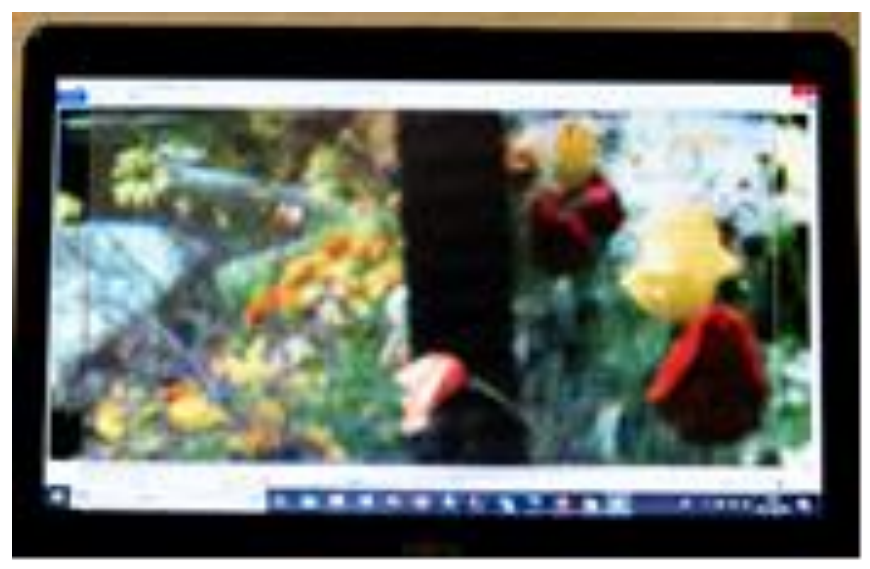

Laptop PC with fly's eye lens

Fig 4: Display of a 3D image 


\section{Conclusion}

A method from synthesizing IP images from raw data captured using a commercially available light field camera developed. Producing appropriate IP images is feasible regardless of the numbers of convex lenses and pixels per lens pitch of IP. The light field camera used in this study is suitable for outdoor and indoor shooting. Moreover, photos previously captured using this light field camera can be converted into IP.

\section{References}

[1] M. G. Lippmann, Epreuves Reversibles Donnant la Sensation du Relief, J. de Phys. , vol. 7, pp. 821 - 825, 1908.

[2] Kazuhisa Yanaka, 2008, Integral photography using hexagonal fly's eye lens and fractional view, Proc. SPIE 6803,Stereoscopic Displays and Applications XIX, 68031K (February 29, 2008), pp. 1-8.

[3] LYTRO meltdown, Retrieved June 20, 2020, from http://optics.miloush.net/lytro/TheCamera.aspx 